\title{
Glomangiopericytoma, three cases in nine days; case reports and Brief review
}

\section{Patrick Stagg MD ${ }^{1^{*}} \mid$ Henry P.Barham MD ${ }^{2,3} \mid$ Rahul V. Ramaraju ${ }^{4}$ | Sai Samyuktha} Bandaru MD ${ }^{1} \mid$ Mohamed A. Taha MD ${ }^{3,5} \mid$ Sanjay Juneja MD ${ }^{1}$

\author{
${ }^{1}$ Baton Rouge General Internal \\ Medicine Residency Program, \\ Baton Rouge, LA, 70809, USA \\ ${ }^{2}$ Rhinology and Skull Base \\ Research Group, Baton Rouge \\ General Medical Center, Baton \\ Rouge, LA, USA \\ ${ }^{3}$ Sinus and Nasal Specialist of \\ Louisiana, Baton Rouge, \\ Louisiana, USA \\ ${ }^{4}$ Department of Chemical \\ Engineering, Louisiana State \\ University, Baton Rouge, LA, \\ 70803, USA \\ ${ }^{5}$ Department of \\ Otorhinolaryngology, Faculty of \\ Medicine, Cairo University, Cairo, \\ Egypt
}

\begin{abstract}
Glomangiopericytoma is a rare sinonasal tumor. We report three cases of this rare disease that presented in nine days to one subspecialty practice. Histochemical and DNA sequencing confirmed the diagnosis in each case. Endoscopic sinonasal surgery provided complete resection in each case. The occurrence of three cases to one practice within a 2-week timeframe may suggest GPC is under-recognized. Routine sequencing to detect CTNNB1 mutations and staining for smooth muscle actin of suspicious sinonasal tumors may increase the frequency of GPC detection.

Copyright : (C) 2022 Medical Editor and Educational Research Publishers
\end{abstract}

\section{1 | INTRODUCTION}

$\mathrm{H}$ emangiopericytomas (HPC) are vascular tumors originating from modified smooth muscle cells, Zimmerman's pericytes. HPC was first described in 1942 by Stout and Murray (1). Sinonasal hemangiopericytomas are clinically and pathologically distinct from hemangiopericytomas (HPC) (2). Compagno and Hyams first described this distinct sinonasal tract tumor in 1976 as "hemangiopericytoma-like" (3). In 2005, sinonasal hemangiopericytoma was reclassified as gloman giopericytoma (GPC) by the World Health Organization. In addition, HPC is now reclassified as solitary fibrous tumors by the WHO. GPC belongs to the category of borderline/low-malignant potential

Supplementary information The online version of this article (10.52845/JORR/2022/3.1.1) contains supplementary material, which is available to authorized users.

Corresponding Author: M. Patrick Stagg $M D$ Baton Rouge General Internal Medicine Residency Program, Baton Rouge, LA, 70809, USA 


\section{GLOMANGIOPERICYTOMA, THREE CASES IN NINE DAYS; CASE REPORTS AND BRIEF REVIEW}

tumors of the sinonasal tract. HPC and glomus tumors are similar tumors that rarely occur in the sinonasal tract but should be in the differential diagnosis when diagnosing GPC.

On clinical presentation, GPC is accompanied most frequently by epistaxis, nasal obstruction, and headache. The lesions are polypoid with an average size of 3.0 to $8.0 \mathrm{~cm}$. They are non-translucent, beefy red to grayish pink, soft, edematous, and fleshy to friable tumors (4). The median age is the seventh decade: 60 years range of 22 to 86 (5), and 62.7 years with a range of in utero to 86 (4). The usual treatment for GPC is endoscopic sinonasal surgery. A few reports of GPC deaths exist, but completely resected disease seldom recurs.

Pathologically GPC is distinguished from soft tissue hemangiopericytoma by the typical diffuse reactivity for actin, factor XIIIA, and vimentin without intense diffuse staining for CD34 (6). B-catenin nuclear accumulation is a diagnostic marker for GPC (7). CTNNB1 mutations lead to nuclear accumulation of B-catenin (5) , (7) , (8) and distinguish GPC from HPCs. Nuclear expression of cyclin D1 is present in tumors with nuclear expression of B-catenin. GPC accounts for less than 0.5 to $1 \%$ of all sinonasal tumors. One of the most significant numbers reported is one hundred four cases of sinonasal-type hemangiopericytoma diagnosed between 1970 and 1995 from the Armed Forces Institute of Pathology files (9). A systematic review in English identified one hundred and ninety-four cases of sinonasal hemangiopericytoma (10). A systematic review in English and Korean identified 337 cases (11). The authors concluded that Actin and CD34 immunostaining could be independent prognostic indicators of GPCs. CTNNB1 mutational analysis became available after 2000 . The most extensive series reported of CTNNB1 mutation confirmed GPC is twenty-three cases (5). Here we report three confirmed cases of GPC with CTNNB1 mutations diagnosed in the same subspecialty practice within nine days. All three cases underwent complete resection by endoscopic sinus surgery (ESS).

\section{2 | PATIENT 1}

A 52-year old Caucasian male with a three-year history of progressive nasal congestion, postnasal drip, and facial pressure was evaluated by his ENT physician in May 2021. He had years of antibiotics, nasal steroid sprays, and antihistamines without improvement. A CT scan of the paranasal sinuses at an outside facility in May 2021 revealed a left sphenoid mass and opacified sinuses. His endoscopic examination here by Dr. Barham revealed a vascular appearing mass in his left olfactory cleft and the right anterior face of the sphenoid. Initially, this mass was suspected to be olfactory neuroblastoma. The patient underwent endoscopic sinus surgery with bilateral maxillary antrostomy and complete resection of the sinonasal mass in July 2021. The frozen section was consistent with a small round blue cell tumor. Histopathological examination of specimens showed neoplastic proliferation of cells with signs of vesicular and storiform formation with a meningothelial appearance. Immunohistological analysis showed positive staining for $\beta$-catenin, smooth muscle actin, S-100, and a Ki-67 index of 5\%. The tumor cells were negative for Synaptophysin, S100, Melan A, Desmin, P40, AE1/3, CK8/18, SMMS1, CD56, Myogenin, P63, SOX-10, and Chromogranin. Next-generation Genetic sequencing and analysis showed a CTNNB1 DNA alteration (c.98 C $>\mathrm{G}$ ) and the alternation of the corresponding protein (p.533C at exon 3 ). The tumor is a glomangiopericytoma with positive staining for $\beta$-catenin, smooth muscle actin, and the presence of the CTNNB1 mutation.

\section{3 | PATIENT 2}

A 58-year old Caucasian male began having recurrent epistaxis, sinus congestion, facial pressure, left-sided nasal obstruction, and a decreased sense of smell for the last nine months. He had antibiotics, nasal steroid sprays, and antihistamines without improvement in symptoms. In October 2020, he presented to the hospital for epistaxis, which required cauterization in the operating room. A lesion was identified clinically as possible neuroblastoma. He 


\section{MEDICAL EDITOR AND EDUCATIONAL RESEARCH}

saw Dr. Barham for further evaluation in June 2021, and a CT paranasal sinus scan showed a left olfactory cleft mass attached to the skull base. He underwent endoscopic excision of the sinonasal and skull base mass with a skull base reconstruction in June 2021. Histopathological examination revealed neoplastic architecture and a storiform/whorled configuration of tumor cells separated by a vascular pattern; the cells were closely packed, uniform, and showed oval nuclei. Immunohistological analysis showed positive staining for $\beta$-catenin, smooth muscle actin. The tumor cells were negative for Synaptophysin, S100, Melan A, Desmin, P40, AE1/3, CK8/18, SMMS1, CD56, Myogenin, P63, SOX-10, and Chromogranin. Genetic sequencing analysis showed CTNNB1 DNA alteration $(\mathrm{c} 98 \mathrm{C}>\mathrm{T})$ and protein alteration $(\mathrm{p} 533 \mathrm{~F}$ at exon 3 DNA alteration c. $98>\mathrm{G})$. The tumor is a glomangiopericytoma with positive staining for $\beta$-catenin, smooth muscle actin, and the presence of the CTNNB1 mutation.

\section{4 | PATIENT 3}

A 65-year old Caucasian female with a two-year history of nasal congestion, obstruction, epistaxis, and shortness of breath saw her ENT physician. She had a past medical history of Insulin-dependent Diabetes Mellitus, Hypertrophic Obstructive Cardiomyopathy, TIA, COPD, and Meniere's disease. She received nasal sprays, steroids, antibiotics, antihistamines, none of which resulted in any longterm improvement in symptoms. The patient had imaging at an outside facility that showed a sinonasal mass. Partial resection occurred at an outside facility in April 2021. Pathology reported a hemangiopericytoma. The patient had a recurrence of her symptoms and saw Dr. Barham for further evaluation in May 2021. A CT scan of the paranasal sinuses performed at Baton Rouge General Hospital showed a left sinonasal mass with skull base involvement and a left maxillary polyp. She underwent endoscopic sinus surgery with complete excision of the sinonasal tumor, maxillary antrostomy, left ethmoidectomy, left sphenoidotomy, and excision of skull base tumor with reconstruction in July 2021. Histopathological examination revealed storiform formation of tumor cells. Immunohistological analysis showed positive staining for Factor XIIIa and smooth muscle actin. The tumor cells were negative for CD34 and BCL-2. Genetic sequencing and analysis were performed and showed a CTNNB1 DNA alteration (c.98 C $>$ G) and the alternation of the corresponding protein (p.533C at exon 3). The tumor is a glomangiopericytoma with positive staining for smooth muscle actin and a CTNNB1 mutation.

\section{5 | DISCUSSION}

The WHO defines GPC as a sinonasal tumor demonstrating a perivascular myoid phenotype (smooth muscle actin-positive); It is a borderline and low malignant potential soft tissue tumor of the nose and paranasal sinuses (4) . GPC represents less than $0.5 \%$ of all sinonasal neoplasms with a peak incidence in the seventh decade. The common presenting symptoms are unilateral nasal obstruction and recurrent epistaxis. Less common findings include headache, pain, difficulty in breathing. GPC is polypoid, beefy red to grayish pink, soft, fleshy to friable, and edematous to hemorrhagic appearance, which easily bleeds on clinical examination (12). Histologic examination is necessary for the diagnosis. Hematoxylineosin staining reveals subepithelial well-circumscribed, unencapsulated cellular tumor, characterized by diffuse closely packed cells, forming short fascicles and exhibiting storiform, whorled, or palisaded pattern, interspersed with many thin-walled, branching staghorn vessels. The neoplastic cells are uniform and elongated to oval with a round to spindle-shaped nuclei and eosinophilic cytoplasm (12). Glomangiopericytoma also has limited mitotic activity, atypia. It is distinguished from soft tissue hemangiopericytoma by the typical diffuse reactivity for actin, factor XIIIA, and vimentin without intense diffuse staining for CD34] (9).

B-catenin is a key regulatory molecule of the Wnt signaling pathway, which is essential for tissue homeostasis and regulation of cell proliferation, differentiation, and function. B-catenin connects "on " and "off" states of Wnt signaling to the transcription process. Abnormal stabilization and nuclear accumulation of B-catenin due to missense mutations 


\section{GLOMANGIOPERICYTOMA, THREE CASES IN NINE DAYS; CASE REPORTS AND BRIEF REVIEW}

or alternate molecular mechanisms occur at a high frequency in various epithelial cancers (13). In GCT, the gain of function mutations in the gene CTNNB1 (cadherin-associated protein) that encodes B-catenin is likely to initiate molecular events in GPC tumorigenesis (8). Multiple mutations in GPC tumors of the CTNNB1 gene have been described (5), (8). Nuclear accumulation of B-catenin is a diagnostic marker for GPC (7). Therefore, sequencing of GCT lesions seems likely to become a standard of care for definitive diagnosis. Sequencing should allow differentia-tion of GPC from sinonasal solitary fibrous tumors and sinonasal glomus tumors. Recurrent transloca-tions involving identified molecular drivers NAB2 and STAT6 occur in solitary fibrous tumors (14), (15). Glomus tumors demonstrate a gene fusion involving MIR143 with Notch-1, NOTCH-2, or NOTCH-3. MIR143 and NOTCH genes involved vascular smooth muscle development and smooth muscle differentiation (16) .

Endoscopic endonasal tumor resection is the preferred option for GPC tumors as it is less inva-sive, has low morbidity, and has equal outcomes. Complete surgical resection of the tumor results in an excellent overall survival rate. Incomplete tumor resection can result in recurrence (17) . Ad-junct chemotherapy/radiotherapy has limited efficacy when complete surgical resection is not pos-sible (11). We reported three confirmed cases of GPC successfully treated with complete resection by Endoscopic Sinus surgery. A CTNNB1 mutation and the presence of smooth muscle actin are present in all 3 cases. The occurrence of three cases within a two week timeframe in one subspecialty practice may suggest GPC could be more common than recognized. It is possible that routine sequencing of suspicious sinonasal tumors for CTNNB1 mutations and staining for smooth muscle actin may increase the frequency of GPC detection.

\section{REFERENCES}

1. Stout AP, Murray Mr. Hemangiopericytoma: a vascular tumor featuring Zimmermann's pericytes. Ann surg. $1942 \mathrm{Jul} ; 116(1): 26-33$. Doi: $\quad 10.1097 / 00000658-194207000-00004$.
Pmid: 17858068; pmcid: pmc1543753.

2. Dandekar M, Mchugh JB. Sinonasal glomangiopericytoma: case report with emphasis on the differential diagnosis. Arch Pathol Lab Med. 2010;p. 134-134.

3. Compagno J, Hyams VJ. Hemangiopericytomalike intranasal tumors. A clinicopathologic study of 23 cases. Am J Clin Pathol. 1976;66(4):970369-970369.

4. Thompson, Lester D R, and Julie C FanburgSmith. "Update on Select Benign Mesenchymal and Meningothelial Sinonasal Tract Lesions." Head and neck pathology vol. 10,1 (2016): 95 108. doi:10.1007/s12105-016-0697-6.

5. Kono M, Bandoh N, Matsuoka R, Goto T, Akahane T, Kato Y, et al.

6. Thompson LD, Fanburg-Smith JC, Wenig BM, Barnes L, Eveson JW, Reichart P, et al. Borderline and low malignant potential tumours of soft tissues. In: Pathology and Genetics of Head and Neck Tumours. IARC Press; 2005. p. 43-45.

7. Lasota J, Felisiak-Golabek A, Aly FZ, Wang ZF, Thompson LD, Miettinen M. Nuclear expression and gain-of-function $\beta$-catenin mutation in glomangiopericytoma (sinonasal-type hemangiopericytoma): insight into pathogenesis and a diagnostic marker. Mod Pathol. 2014;28(5):715-720. doi:10.1038/modpathol.2 014.161.

8. Haller F, Bieg M, Moskalev EA, Barthelmeß $\mathrm{S}$, Geddert $\mathrm{H}$, Boltze $\mathrm{C}$, et al. Recurrent mutations within the amino-terminal region of $\beta$ catenin are probable key molecular driver events in sinonasal hemangiopericytoma. Am J Pathol. 2014;185(2):563-571. doi:10.1016/j.ajpath.201 4.10.019.

9. Thompson LD, Miettinen M, Wenig BM. Sinonasal-type hemangiopericytoma: a clinicopathologic and immunophenotypic analysis of 104 cases showing perivascular myoid differentiation. Am J Surg Pathol. 2003;27:737-749. do i:10.1097/00000478-200306000-00004. 


\section{MEDICAL EDITOR AND EDUCATIONAL RESEARCH}

10. Duval M; 2012. doi:10.1002/hed.23074.

11. Park Eun Su, Kim Jiyoung, Jun Sun-Young. Characteristics and prognosis of glomangiopericytoma: a systematic review. Head Neck. 2017:1-13. [PubMed] [Google Scholar].

12. Nunnery EW, Kahn LB, Reddick RL. Hemangiopericytoma: a light microscopic and ultrastructural study. Cancer. 1981;47.

13. Agaimy A, Barthelmeß S, Geddert H, Boltze C, Moskalev EA, Koch M, et al. Phenotypical and molecular distinctness of sinonasal hemangiopericytoma compared to solitary fibrous tumour of the sinonasal tract. Histopathology. 2014;65(5):667-673. doi:10.1111/his.12452.

14. Robinson DR, Wu YM, Kalyana-Sundaram S, Cao X, Lonigro RJ, Sung YS, et al. Identification of recurrent NAB2-STAT6 gene fusions in solitary fibrous tumor by integrative sequencing. Nat Genet. 2013;45(2):180-185. doi:10.1038/n g.2509.

15. Chmielecki J, Crago AM, Rosenberg M, Connor O, Walker R, Ambrogio SR, et al. Whole-exome sequencing identifies a recurrent NAB2-STAT6 fusion in solitary fibrous tumors. Nat Genet. 2013;45(2):131-132. doi:10.1038/ng.2522.

16. Mosquera JM, Sboner A, Zhang L, Chen CL, Sung YS, Chen HW, et al. Novel MIR143$\mathrm{NOTCH}$ fusions in benign and malignant glomus tumors. Genes Chromosomes Cancer. 2013;52(11):1075-1087. doi:10.1002/gcc.2210 2.

17. Dandekar M, Mchugh JB. Sinonasal glomangiopericytoma: case report with emphasis on the differential diagnosis. Arch Pathol Lab Med. 2010;p. 134-134.

How to cite this article: M. PATRICK STAGG MD ET AL. Glo-mangiopericytoma, three cases in nine days; case reports and Brief review. Journal of Otolaryngology and Rhinology Research. 2022; 65-69.https://doi.org/10.52845/JORR/2022/3.1.1 